\title{
ESTUDIO EXPLORATORIO Y DESCRIPTIVO DE SEGMENTAR- APUNTAR-POSICIONAR SAP EN MARKETING SOCIAL
}

\author{
EXPLORATORY AND DESCRIPTIVE STUDY OF SEGMENT- \\ POINT-POSITION SAP IN SOCIAL MARKETING
}

\author{
Roberto M. Campos T.' Miguel A. Bustamante U.'
}

'Facultad de Economía y Negocios. Universidad de Talca· Dos Norte ${ }^{685,3465548}$ Talca. Chile. E-mail: rcampos@utalca.cl; mabu@utalcacl

El objetivo principal de esta investigación es un estudio exploratorio del Marketing Social y del S.A.P. (segmentar-apuntar-posicionar), su conceptualización y evolución, proporcionar una guía resumida de la evolución del concepto de marketing social, resaltando los aspectos más importantes. También presenta algunas experiencias de organizaciones en relación a la utilización del S.A.P en Marketing Social. En cuanto al diseño y metodología, el documento es una revisión de información secundaria, revisiones sistemáticas y estudios primarios de intervenciones de mercadeo social, de las cuales se proceden a seleccionar y ordenar en registros, mediante la jerarquización e importancia. Para esto se utiliza el método histórico, con un enfoque exploratorio, descriptivo y cualitativo. La investigación permite concluir que se observa un contexto teórico dinámico y evolutivo en lo que respecta al Marketing Social. Lo que se inició como un concepto de Marketing acotado y de dependencia del marketing comercial, se fue ampliando, y ha evolucionado hacia un sistema estructurado, extenso e integral, modelado y de amplias perspectivas de aplicación, como en temas de sustentabilidad ambiental, social, cultural, y de la satisfacción. Seguido de implicaciones que dichas intervenciones tiene sobre el comportamiento ético de la sociedad. Se convirtió en un proceso que abarca las organizaciones privadas con fines y sin fines de lucro, y entidades públicas. En relación a las aplicaciones de marketing Social y S.A.P., podemos señalar que si bien es cierto en general han sido efectivas, principalmente las relacionadas con la salud pública, educación, política y sociedad. Sin embargo se aprecian contradicciones en los resultados, los cuales pueden deberse a distorsiones en la comprensión del mensaje o debido a problemas actitudinales del receptor, o por la posibilidad de que grupos sociales no objetivo queden afuera de las campañas y a veces ellos también podrían ser beneficiados.

Palabras clave: Marketing Social, Segmentación, responsabilidad social y marketing, aplicaciones de SAP.
The main objective of this research is an exploratory study of Social Marketing and S.A.P. (Segment-pointposition), its conceptualization and development, provee a summary of the evolution of the concept of social marketing guide, highlighting the most important aspects. It also presents some experiences of organizations in relation to the use of S.A.P in Social Marketing. In terms of design and methodology, the document is a review of secondary information, systematic reviews and primary studies of social marketing interventions, of which proceed to select and order in registers, by prioritization and importance. For this the historical method, with an exploratory, descriptive and qualitative approach was used. The research supports the conclusion that a dynamic and evolving theoretical context is observed with regard to Social Marketing. What started as a marketing concept limited and dependence of commercial marketing, it was extended, and has evolved into a structured, extensive and comprehensive modeling and broad application prospects, such as in environmental, social sustainability system, cultural and satisfaction. Followed by implications that such interventions have on the ethical behavior of society. It became a process involving private organizations and purposes non-profit, and public entities. Regarding Social marketing applications and S.A.P., we can say that if it is true in general have been effective, mainly those related to public health, education, politics and society. However contradictions can be seen in the results, which may be due to distortions in understanding the message or because of attitudinal problems of the recipient, or the possibility that social groups do not target left out of campaigns and sometimes they could also be benefited.

Keywords: Social Marketing, Segmentation, social responsibility and marketing, SAP applications. 


\section{Introducción}

El presente paper está diseñado, en base a lo solicitado por la clase de Marketing de Negocios, del programa PhD en Administración de Empresas, de la Université Libre des Sciences del Entreprise et des Technologies de Bruxelles, Bélgica, y EGEU Business School.

En la primera parte del paper se presentan los basamentos teóricos, referidos al S.A.P. (Segmentar - Apuntar - Posicionar) y del Marketing Social, presentando aspectos como: conceptos y evolución. En la segunda parte, se presenta la aplicación del SAP en Marketing Social, algunas experiencias de Organizaciones en Chile y programas internacionales y nacionales.

En relación a los aspectos teóricos, el Marketing Social comprende los esfuerzos por cambiar el comportamiento público, por otro que la sociedad estime deseable (Kotler P. , exponews, 2011). Y el S.A.P. es el proceso estratégico por medio del cual una empresa, puede aumentar la probabilidad de éxito en el lanzamiento de nuevos productos (Weinreich, 2007).

En relación a la metodología para este trabajo, se realiza una recopilación de información de fuentes secundarias, las cuales se proceden a seleccionar y ordenar en registros, mediante la jerarquización e importancia que tengan para el artículo, y particularmente lo relacionado con el Marketing Social y S.A.P. Para esta fase se utiliza el método histórico, para describir la evolución de los aspectos más importantes de este estudio, así como también un enfoque exploratorio, descriptivo y cualitativo, con el fin de dar a conocer los fenómenos que han logrado el desarrollo cada vez más creciente del S.A.P. y del Marketing Social.

El fin del artículo es un estudio exploratorio y descriptivo del Marketing Social y el S.A.P., su conceptualización y evolución, proporcionar al lector una guía resumida de la evolución del concepto de marketing social, resaltando los aspectos más importantes, así como la utilización del S.A.P. en el Marketing Social, también presentar algunas experiencias de organizaciones con respecto al Marketing Social, y en relación a la utilización del S.A.P., en Chile. Conscientes de nuestras limitaciones, proporcionamos nuestras interpretaciones descriptivas, basadas en las lecturas y recopilaciones, impulsados por la necesidad de una mayor conciencia científica sobre el tema.

\section{Conceptos de Marketing}

El concepto de Marketing, es el proceso mediante el cual las compañías crean valor para los clientes y establecen relaciones sólidas para obtener a cambio valor de ellos (Kotler \& Armstrong, 2012). Es la "Filosofía según la cual, el logro de las metas de la organización, depende de conocer las necesidades y los deseos de los mercados meta, así como de proporcionar las satisfacciones deseadas de mejor manera que los competidores" (Kotler \& Armstrong, Marketing, 2012). Por otra parte, el marketing social, es el uso de conceptos y herramientas de marketing comercial, en programas diseñados para influir en el comportamiento de los individuos, con el fin de incrementar su bienestar y el de la sociedad (Kotler
\& Armstrong, Marketing, 2012). Kotler señala que, el Marketing Social comprende los esfuerzos por cambiar el comportamiento público, por otro que la sociedad estime deseable (Kotler P. , exponews, 2011). Por otra parte, "El Marketing Social no es una ciencia, sino un arte profesional que se basa en múltiples disciplinas científicas, para crear programas, diseñados para influir en el comportamiento humano a gran escala" (Smith, 2006, p. 38).

Así mismo, "El Marketing Social es la aplicación de las técnicas del marketing comercial para el análisis, planteamiento, ejecución y evaluación de programas diseñados, para influir en el comportamiento voluntario de la audiencia objetivo, en orden a mejorar su bienestar personal y el de su sociedad" (Andreasen A. , 1995), "la aplicación sistemática de los conceptos y técnicas de marketing, para lograr los objetivos específicos de comportamiento, para un bien social o público" (Craig Lefebvre, 2011, p. 56). El Marketing Social facilita la aceptación, rechazo, modificación, abandono, o el mantenimiento de determinadas conductas, por grupos de individuos, que a menudo son descritos como el público objetivo.

La definición de Marketing Social, también incluye el análisis de las consecuencias sociales, de las políticas y actividades del marketing comercial, por ejemplo el seguimiento de prácticas de marketing en empresas como la del tabaco, alimentación, bebidas alcohólicas, etc. (Greir \& Bryant, 2005). Este proceso de Marketing Social: "es un proceso continuo e iterativo, y que consta de seis grandes pasos o tareas: planificación inicial; investigación formativa; desarrollo de estrategias; desarrollo de programas y pruebas preliminares de las intervenciones materiales y no materiales; implementación; monitoreo y evaluación” (Greir \& Bryant, 2005, p. 326)

"El Marketing Social se ha beneficiado sin duda, de la transferencia de conocimientos del Marketing Comercial" (Newton, Newton, Turk, \& Ewing, 2013, p. 1422). Sin embargo, existen diferencias entre el Marketing Comercial y el Marketing Social, que hacen que la transferencia sistemática de técnicas de un campo a otro, sea potencialmente problemático (Newton, Newton, Turk, \& Ewing, 2013). Al respecto, por ejemplo la planeación, es parte relevante de toda organización y aunque por lo general las ONG, asociadas a programas de Marketing Social sean fundadas por motivos personales, es importante que noolviden ésta parteimportante dela administración, ya que si el segmento al que quieren atender, no es suficientemente grande, será difícil que los donadores, quieran darles dinero, porque preferirán entregarlo a causas que tengan más necesidad y sean más estructuradas administrativamente. También el concepto de Marketing Social cuestiona, si la concepción pura de Marketing pasa por alto, conflictos posibles entre los deseos a corto plazo del consumidor y el bienestar a largo plazo del mismo (Kotler \& Armstrong, Marketing, 2012).

El Marketing Social "exige un Marketing sustentable, es decir, un marketing responsable a nivel social y ambiental, que cubra las necesidades actuales de los consumidores y de los negocios, pero que al mismo tiempo conserve o mejore la capacidad de las generaciones futuras para cubrir sus necesidades" (Kotler \& Armstrong, Marketing, 2012). Es el Marketing sustentable de una mayor Responsabilidad Social, que es el comportamiento ético de las organizaciones (Bravo, 2007), es “...una 
forma elevada de relacionarse con los demás, es crear sinergias que conduzcan a aumentar el bien común de corto y largo plazo en armonía con el entorno y los intereses comunes e individuales, tácitos y explícitos (Bravo, Responsabilidad Social RS La nueva riqueza de las naciones, 2007). Al respecto, el Marketing Holístico, incorpora el Marketing con Responsabilidad Social (RS) y con ello coloca un elevado énfasis de preocupaciones amplias, en el contexto ético, ambiental, legal, social, de las actividades y programas de Marketing (Carasila, 2008).

Así mismo, la Responsabilidad Social es practicar y fomentar el comportamiento ético, en especial, hacernos cargo de todas nuestras formas de influencia sobre las personas y el medio ambiente" (Bravo, 2007, p. 26), es en este sentido que se relaciona con el Marketing Social. La ética corporativa y la Responsabilidad Social, se han convertido en temas candentes en casi cualquier negocio (Kotler \& Armstrong, Marketing, 2012). Por otra parte el Marketing Social "en su concepto, hace énfasis en la calidad de vida y en la forma como se deben modificar las prácticas, conductas, comportamientos, actitudes y creencias, que pueden resultar nocivas para la sociedad, y de esta manera, incorporar dentro del mercadeo la necesidad de relacionar la marca de un producto o servicio, con causas sociales con las cuales se identifica y se compromete a transformar" (Muñoz, 2001, p. 38). Por esta razón, la responsabilidad social se ha transformado en una nueva forma de comunicarse con los consumidores y públicos de interés. Surge así el concepto y aplicación del Marketing con causa o Marketing Social (Garrido, Winicki, Vidal, Urquieta, \& Pinto, 2015).

\section{Marketing, estrategia y segmentación}

Con respecto al ámbito estratégico de las organizaciones, el concepto de Marketing Social, es el principio de Marketing ilustrado, que establece que una compañía, debería tomar buenas decisiones de Marketing, considerando los deseos de los consumidores, los requerimientos de la compañía, así como también los intereses a largo plazo de los consumidores y de la sociedad (Kotler \& Armrstrong, Marketing Versión para Latinoamerica, 2007). "El concepto de Marketing Social, también señala que la estrategia de Marketing debería entregar valor para los clientes, de forma que conserve o mejore el bienestar tanto del consumidor como de la sociedad" (Kotler \& Armrstrong, Marketing Versión para Latinoamerica, 2007, p. 11). En este sentido, la clave para un buen Marketing Social, está en hablar con (y escuchar a) la gente a la que usted desea llegar. Se trata de un proceso orientado hacia el cliente, por ello, todos los aspectos de su programa deben ser desarrollados con los deseos, necesidades y carencias del público objetivo como foco central (Kline Weireich, 2001).

En relación al concepto de S.A.P. que es: Segmentar, Apuntar y Posicionar, podemos señalar que es "el proceso por medio del cual se aumenta la probabilidad de éxito en el lanzamiento de nuevos productos" (Weinreich, 2007, p. 56). El concepto de S.A.P. contiene las etapas de: "Segmentar donde se debe (1) Identificar variables de segmentación y partir el mercado en segmentos, (2) Desarrollar perfiles de los segmentos o mercados meta. Apuntar donde (1) Hay que evaluar la atractividad de los segmentos (2) Seleccionar los segmentos y los mercados meta y Posicionar (1) Identificar posibles conceptos de posicionamiento del mercado meta escogido ¿Cómo quiero que me identifiquen? Y (2) Definir el producto a ofrecer, a que precio y cómo comunicarlo efectivamente" (Weinreich, 2007, p. 6o).

Por otra parte, el concepto de segmentar un mercado, es el proceso de dividir un mercado en distintos grupos de compradores, con necesidades, características o conducta diferentes, y quienes podrían requerir productos o programas de marketing separados (Kotler \& Armstrong, Marketing, 2012).”Un segmento de mercado, es un grupo de consumidores, que responde de manera similar, a un conjunto dado de actividades de Marketing "(Kotler \& Armstrong, Marketing, 2012, p. 49). Es la necesidad de dividir al mercado en grupos, cuyos integrantes posean características homogéneas. "El principio fundamental del Marketing, es que es imposible satisfacer a todo tipo de clientes con un solo producto o servicio para todos igual" (Weinreich, Segmentación de Mercados. Conceptos, propósitos y metodología, 2016).

Al respecto la segmentación de mercados sociales, es la necesidad de dividir su audiencia en grupos de acuerdo a criterios particulares. Los miembros de cada grupo tienen al menos un factor importante en común, con respecto a los otros miembros del mismo grupo, ese factor los distingue de los otros grupos (Juárez, 2015). Se pueden segmentar por el ámbito socio demográfico (edad, sexo, cultura, nivel social, religión), psicográfico (personalidad, estilo de vida, gustos personales), geográficas (ubicación geográfica, ámbito local-global, delimitación por zonas), variables de conducta (beneficio buscado, conveniencia, decisión de compra, riesgo percibido, actitud, motivación, expectativas), o epidemiológicas en programas de Marketing Social dirigido a la salud pública. Otros aspectos importantes con respecto a la segmentación son: la preocupación por el costo, la disposición a arriesgarse al cambio del grupo objetivo, los beneficiarios (personas que reciben el beneficio social), identificación y accesibilidad a los beneficiarios (Juárez, 2015).

Análogamente con respecto al mercadeo social, es importante señalar y determinar el mercado meta, grupo social objetivo o target market, que es el destinatario ideal de una determinada campaña, producto o servicio. La selección de clientes o grupo social a los que se quiere llegar. Es decir "Apuntar" a un mercado meta, ese mercado meta implica, la evaluación del atractivo de cada segmento del mercado y la elección de uno o más segmentos para ingresar en ellos (Kotler \& Armrstrong, Marketing Versión para Latinoamerica, 2007).

Con respecto a los programas de Marketing, las organizaciones sociales deben tomar en cuenta, los nichos en los que se cree que el programa social va a ser más fructífero, esto se hace mediante la elaboración del perfil de la población objetivo. Así también es relevante determinar el perfil de los donadores, complementadores, las personas y organizaciones internacionales que apoyan a los programas sociales de la localidad. Sin embargo, lo principal de realizar esta segmentación, es investigar la magnitud del segmento, también será es importante saber qué tan necesaria es nuestra ayuda. Sin embargo cabe señalar, que muchas veces al segmentar el mercado social, se deja afuera a grupos que también necesitaban del programa, es una de las criticas que se le hacen a la 
segmentación en el Marketing Social. Así, mientras algunos grupos, que también pueden beneficiarse de la exposición a una campaña de Marketing Social, en última instancia, pueden ser excluidos de la recepción de dicha campaña (Newton, Newton, Turk, \& Ewing, 2013).

\section{Marketing y posicionamiento}

El posicionamiento significa, hacer que un producto ocupe un lugar claro, distintivo y deseable en la mente de los consumidores meta, en relación con los productos competidores o grupo social objetivo. Dentro del grupo objetivo, las personas deben ser identificadas por sus diferentes necesidades sociales y el posicionamiento según los beneficios esperados. Con respecto a la accesibilidad a las personas, debe ser posible alcanzar y servir al segmento a través de la comunicación con medios y soportes adecuados (Juárez, 2015). El segmento debe ser estable a través del tiempo, con lo que los programas de medios, no pueden ser reemplazados continuamente, es decir, deberían ser sostenibles a mediano y largo plazo. Así mismo, en relación a las personas, este voluntariado que atenderá al segmento, es un conjunto de personas que se unen libre y desinteresadamente al programa, para fines benéficos y altruistas, y son importantes en las campañas de cambio social (Juárez, 2015). Ya que según Kotler (1992) el Marketing Social son estrategias para cambiar la conducta pública (Kotler P. , exponews, 2011).

En relación a este posicionamiento, es relevante lo siguiente: Donde se combina el producto, el servicio o idea a promover con un precio adecuado; eliminar o minimizar las barreras para su accesibilidad; inducir su adquisición y considerar siempre los diferentes públicos o grupos meta. Si se obtiene la combinación adecuada de todos los componentes, se logra el posicionamiento del producto, servicio o idea, en Marketing Social (Suárez Lugo, 2013). Habitualmente un indicador referente del posicionamiento en productos o servicios son las ventas, en el caso social, que es de ideas, de conocimientos, de opiniones y actitudes, el indicador más representativo es la conducta (Suárez Lugo, 2013).

Así el mercadeo social, se establece como un instrumento de ideas, actitudes, comportamientos y situaciones socialmente consideradas, como problemas sociales (Moliner, 1998). Al respecto las causas sociales, desde el punto de vista del marketing social, y su posicionamiento, se puede vincular a diversos sectores de la sociedad, como también a sectores productivos, tales como las empresas privadas y entidades públicas de toda clase, también organismos no gubernamentales. Además del enfoque de cada uno de los actores, denominados agentes de cambio, quienes combaten o enfrentan diversas problemáticas tales como: la pobreza, la drogadicción, la ecología, la responsabilidad social, la protección infantil, la economía y la producción, el consumo de producto nacional, el contrabando, la salud preventiva, etc. (Aristizabal Botero, Restrepo Múnero, \& Arias Pérez, 2007).

\section{Evolución del Marketing Social y del S.A.P.}

En relación a la evolución que ha tenido Marketing Social, podemos señalar, que hace algunos años, es que aparece el término de mercadeo social en el marco de un esquema de rentabilidad, ventas y generación de beneficios, y que promueve la humanización, por medio de la promoción de causas sociales, cambios de actitudes, así como también la transformación de prácticas, que se encuentran en un desarrollo sostenible y responsable. Para algunos era tomado como una práctica no ortodoxa del mercadeo tradicional, que afectaba y era afectado por las normas éticas de la sociedad. (Kotler \& Zaltman, Social Marketing : An Approach to Planned Social Change Journal of Marketing, 1971), esto ahora tiene un gran sentido, de acuerdo a la evolución de los mercados globales, en donde se ha evolucionado hacia a un tipo de oferta mayor que la demanda de productos y servicios.

El marketing social ha estado en la literatura de marketing desde la década de 1960 , las variaciones de mercadeo social se han aplicado tanto para promover la seguridad del tráfico, el control del tabaco, la prevención de drogas, las vacunas infantiles, la mejora de la nutrición y la dieta, y el comportamiento del medio ambiente. Así como también para reducir la mortalidad infantil (Smith, 2006). Al respecto, se puede decir que el Marketing Social, es una intervención de carácter "ampliado" (Smith, 2006).

En 1965 la Universidad de Ohio, definió el Marketing, como el proceso por el cual una "Sociedad" anticipa, aplaza o satisface la estructura de la demanda de bienes y servicios económicos, mediante la concepción, la promoción, el intercambio y la distribución física de bienes y servicios (Carasila, 2008). Esta definición considera claramente el marketing como un proceso social y amplio, provocando reacciones y discusiones intelectuales, en relación al dilema de que sí el marketing como tal, debe incluir o tratar los intercambios sociales o los llamados aspectos no lucrativos.

En 1969 Stanton reflexiona sobre el marketing en general y lo define como un sistema de actividades empresariales encaminado a planificar, fijar precios, promover y distribuir productos y servicios que satisfacen necesidades de los consumidores actuales o potenciales, con una clara ampliación del campo de acción, incluyendo al social (Stanton, 1969). También podemos citar a Kotler y Levy (1969), que consideran que el concepto de marketing debe ser ampliado, incluyendo a las organizaciones no empresariales, ya que éstas también, venden sus "productos", poseen "consumidores" y utilizan las variables de Marketing (Kotler \& Levy, Broading The Concept of Marketing, 1969). Por otro lado, Lazer en 1969, defiende la dimensión social del marketing, señalando que es algo más que una tecnología al servicio de la empresa (Lazer, 1969). Así mismo las consecuencias sociales del Marketing, planteadas por Lazer y Dawson, que acentuaba la evaluación de la deseabilidad y honestidad de las actividades de marketing, por ello el marketing ecológico y el consumismo podrían ser integrados a esta perspectiva.

En 1972 Philip Kotler, también desarrolla aspectos del Marketing Social, para ello proporciona cuatro axiomas, el primero hace referencia a que el marketing implica dos o más unidades sociales, cada una con dos o más actores humanos, en el segundo axioma, señala que al menos una de las unidades sociales, busca una respuesta específica de otra $u$ otras en relación a algún objeto social. El tercer axioma, sostiene que la probabilidad que el mercado produzca la respuesta deseada no es fija. Finalmente 
el cuarto axioma, afirma que el Marketing, es el intento de producir la respuesta deseada, creando y ofreciendo valores al mercado (Carasila, 2008).

En 1974, Robert Bartels, propone que el Marketing debiera ser ampliado, tanto como para incluir los campos de aplicación lucrativos y no lucrativos (Show \& Tamilia, 2001). En este sentido esto puede lograr, que el marketing renazca con otro nombre, mucho más amplio. (Bartels, 1974). También en 1975 Bagozi, incorpora al Marketing, actividades relacionadas con el intercambio y los fenómenos asociados al Marketing. Se aprecia una ampliación del marketing, estrictamente relacionada con la Responsabilidad Social, con un componente ético relevante, y una expansión importante del Marketing, desde las organizaciones con fines de lucro hacia las no lucrativas, surgiendo y desarrollando el concepto de Marketing Social. Por lo que claramente, es una nueva dimensión del Marketing, un ensanchamiento del horizonte conceptual al campo de las ideas y de las organizaciones no lucrativas, tales como: iglesias, escuelas públicas y museos, entre otros. En la medida en que éstas, poseen productos o servicios que ofrecen a unos clientes, y utilizan herramientas de Marketing (Kotler \& Levy, Broading The Concept of Marketing, 1969).

Se puede señalar que surge el término "Marketing Social”, definido como: el diseño, implementación, y control de programas pensados para influir en la aceptación de ideas sociales e implicando consideraciones de planificación de producto, precio, comunicación, distribución, e investigación de marketing (Kotler \& Zaltman, Social Marketing : An Approach to Planned Social Change Journal of Marketing, 1971). Se establece en consecuencia el rol relevante del Marketing, en el desarrollo y el cambio social, y también del entorno de las organizaciones. Es decir de las organizaciones que produzcan bienes y servicios para un grupo determinado de consumidores, sea o no preciso el pago, con lo cual la disciplina se amplia a servicios públicos (educación, policía, política)

Philip Kotler (1984) sostiene que el Marketing es un proceso social, por el cual los individuos y las organizaciones obtienen lo que desean y necesitan, mediante la creación, el intercambio de productos y servicios con otros (Kotler P. , Marketing Essentials ( later changed to marketing an introduction): Englewoods cliffs, 1984).

En 1989 Grönroos define el Marketing desde un punto de vista más amplio y social, como una disciplina que crea, desarrolla y comercializa relaciones de intercambio con el cliente a largo plazo, de forma que los objetivos de las partes implicadas, se satisfagan. Esto se realiza mediante un intercambio mutuo y mediante el mantenimiento de las promesas. Se puede considerar, que esto, debería constituirse en una definición general de Marketing (Grönroos, 1989).

A partir de 1999 el Marketing Social, se empieza a caracterizar como un situación de relaciones de redes y de interacciones (Gummesson, 1999). Seguidamente, en la década del 200o, el Marketing Social, se ha visto influenciado por el importante desarrollo y crecimiento de la red global Internet, y de las TI (Tecnologías de Información), así tanto las organizaciones comerciales como las no lucrativas, han tenido que adecuarse a estos cambios $y$ expresiones, de estas nuevas herramientas y redes sociales, es donde el Marketing Social, juega un rol relevante, incluso desde el punto de vista estratégico. Kotler ha reconocido la importancia, que tienen estas herramientas para el Marketing en general (Kotler P. , Dirección de Marketing, 200o).

También, se puede señalar la aparición de nuevos mercados, como el mercado digital, el desarrollo de éste (Rayport \& Sviokla, 1995), aquí las redes sociales tienen un rol destacado en el cambio del comportamiento público. La evolución de estos mercados ha llevado a un nuevo paradigma social, Kotler pone la atención en el "valor", también en el ámbito social, en las redes de colaboración, y en el concepto de marketing holístico, como Marketing con Responsabilidad Social (Kotler \& Zaltman, Social Marketing : An Approach to Planned Social Change Journal of Marketing, 1971).

Se puede apreciar la ampliación del concepto de Marketing tradicional, esto debido a los cambios en el entorno, el desarrollo tecnológico, las actividades de exploración de nuevas oportunidades de valor, y en especial en el concepto de "Valor Compartido" dicho por Porter, donde no es suficiente la responsabilidad clásica de los empresarios, ni la filantropía para responder con efectividad a las necesidades sociales (Mutis, 2015). Se debe redefinir el propósito de las empresas, porque de eso depende su progreso y su existencia (Mutis, 2015). En este sentido, es la creación de nuevos ofrecimientos de "valor", el valor definido como la Propuesta de "Valor", o sumatoria de "Beneficios.

Al respecto, los consumidores no compran productos por sus atributos, si no por esa propuesta de valor, o simetría de beneficios, que es el uso o consumo del producto que promete y entrega (Weinreich, Value Management: La Gestión Estratégica de Posicionamiento de Productos ( GEPP), 2016), en este sentido podemos aplicarlo a Marketing Social mediante el valor de los beneficios del servicio, su valorización de satisfacción, por parte del grupo social. Por otra parte, en Marketing Social actualmente, también son relevante: los beneficios de los servicios entregados, los cuales se constituyen en un valor de satisfacción, en el desarrollo de relaciones de largo plazo, mutuamente satisfactorio y una prosperidad compartida (Kotler \& Keller, Marketing Management, 2006).

Los nuevos conceptos de cliente, "valor", relaciones y redes sociales, internet, Stakeholders y marketing holístico, así como también, lo mencionado anteriormente: el marketing digital, el E-Marketing, y las aplicaciones practicas de internet, celulares, TV digital, y herramientas como whatsapp, Facebook, twitter, hashtack. Estos tienen actualmente una real importancia en el desarrollo del Marketing Social. Al respecto, podemos citar que "hemos cambiado la forma de comunicarnos, de búsqueda de información, de entretenernos, y de comprar, se ha creado toda una disciplina "marketera" para dominar, cómo vender a través de la web, cómo promocionar productos a través de la web" (Weinreich, La practica del marketing y su evolución en el tiempo, 2016), y que claramente también inciden, en la gestión del Marketing Social.

En relación a lo expuesto anteriormente, entonces el Marketing Social, es una práctica flexible, ecléctica de cambio social a gran escala. "Es una forma de pensar 
que hace hincapié en un " intercambio de valor " con el público en lugar del control social o educación por sí sola” (Smith, 2006, p. 40).

\section{Evolución del S.A.P. (Segmentar- Apuntar-Posicionar)}

Por otra parte, con respecto al concepto del S.A.P., el proceso de la globalización en los mercados y es en esta circunstancia que para el "empresario de hoy el reto esta en hacer negocios en un ambiente de mercado caracterizado por (a) mercados heterogéneos (b) competencia intensa y en donde (c) la oferta es mayor que la demanda" (Weinreich, 2007).

En los años 70-80, con respecto a la segmentación de mercados, su conceptualización era de que muchos mercados no eran homogéneos, sino que heterogéneos. Así "No todos los compradores tienen las mismas necesidades; algunos buscan ciertos beneficios, y otros, beneficios diferentes" (Weinreich, La practica del marketing y su evolución en el tiempo, 2016, p. 11). Se hace relevante, la necesidad de reconocer segmentos de mercado para "apuntar mejor las baterías". Cómo lo explica el profesor Ph.D. Rolf Weinreich, el Concepto S:A:P de Philip Kotler, "Segmentar, Apuntar, Posicionar, es la tónica del marketing de hoy" (Weinreich, La practica del marketing y su evolución en el tiempo, 2016). En la época de los 9o', están las estrategias competitivas de Michael Porter, las Estrategias Genéricas, para diferenciarse de la competencia y obtener resultados superiores, "o se compite por Valor, o por Precio....no hay de otra" (Weinreich, La practica del marketing y su evolución en el tiempo, 2016, p. 16), las estrategias de Diferenciación (competir por Valor), las Estrategias de Liderazgo en Costos (competir con Precios menores) y la Estrategia de Focalización (las anteriores, pero en un segmento del mercado total). Surgen después otros conceptos, uno de ellos, el Marketing Responsable, que luego se amplió como Marketing Sustentable y que considera, de acuerdo a Donald Fuller, el proceso de planificar, implementar y controlar el desarrollo, precio, promoción y distribución de productos en una forma que considere satisfacer las necesidades de los consumidores y atender las metas de la organización de una manera que sea compatible con el ecosistema.

Actualmente, también es importante para la segmentación, el concepto de "Value Innovation", estrategias de Innovación en el Valor de Kim y Marbougne, cómo enfrentar mercados en que los productos son prácticamente "commodities", cómo reinventar productos o servicios, para competir en mercados no discutidos, estaregia Oceanos azules (Kim \& Mauborgne, 2004). Que son aquellas innovaciones en productos, que hacen cambiar el "rayado de la cancha" en una industria y que definen nuevas pautas de competición. "Formas de ampliar la Demanda con cambios en los Procesos de Producción, Entrega y Cadena de Valor de los Clientes". (Weinreich, La practica del marketing y su evolución en el tiempo, 2016, p. 30). Al respecto en Marketing Social, se aplica con la innovación de los servicios hacia los grupos sociales objetivos, de tal forma que sean más eficaces y más eficientes, en el encuentro del "valor".

\section{Aplicaciones, evidencias del Marketing Social y S.A.P.}

El mercadeo social se centra en las personas, sus deseos y necesidades, aspiraciones, estilo de vida, la libertad de elección (Walsh, Shiu, Graig Andrews, \& Hasting, 2010). Así mismo las personas son únicas, con sus propios valores, creencias y actitudes, sin embargo existen similitudes en relación a sus necesidades y deseos, como también en la forma de expresar su satisfacción, lo que se puede reflejar en las agrupaciones sociales. Por ello aunque todas las personas y las comunidades son diferentes entre sí, se pueden agrupar en segmentos, que maximizan nuestra capacidad de influir en su comportamiento. Un segmento de audiencia, es un grupo de individuos que comparten una serie de características comunes, estas características incluyen: $\mathrm{N}$-demográficos de la misma edad, ingresos, género, étnica o rango $\mathrm{N}$ (Smith, 2006). Se pude decir que existen tres aspectos relevantes de un segmento que lo hacen más viable: a) Su comportamiento, que contribuye a la solución del problema; b) ¿Es el segmento lo suficientemente grande, como para hacer una diferencia medible? ; c) Es el segmento suficiente para nosotros, para llegar de manera efectiva con nuestros recursos (Smith, 2006). Al respecto, un segmento se basa en una combinación de estos factores.

En este sentido las actividades de marketing, como la segmentación, comienzan con un enfoque en la comprensión de la gente, sus deseos y necesidades, sus aspiraciones, el estilo de vida y las opciones que tienen. Por ello el desarrollo y la aplicación de las campañas, que apelan a grupos específicos de personas o consumidores, aumentan la eficiencia y eficacia de la Mercadotecnia Social (Kotler \& Lee, 2008). Es la segmentación, apuntando a grupos de consumidores o beneficiarios (sociales), por ejemplo, los programas de atención psicológica para grupo de adultos mayores (mayores de 65 años) o programas de sexo seguros a mujeres adultas. $O$ por ejemplo una acción observable, en un específico segmento $\mathrm{N}$ de la población, bajo específicas condiciones, ejemplo de ello son los hombres, entre las edades de 17 y 20 , que usarán el cinturón de seguridad, antes de poner el coche en marcha, y cuando conduce un vehículo de pasajeros (Smith, 2006).

Estas técnicas de segmentación, pueden ser un medio eficaz para motivar el cambio de comportamiento, en contextos tan variados como: el aumento de los niveles de actividad física; la prevención del tabaquismo; campañas de no al alcoholismo o de sexo seguro. Al respecto, un punto de discusión relevante, es por ejemplo la aplicación de la segmentación y posicionamiento de campañas de salud, de sexo seguro en mujeres adultas, que se segmentan sin contemplar como grupo objetivo a las mujeres mayores, en este caso éstas últimas no suelen ser el blanco u objetivo de las campañas, ya que su riesgo histórico de contraer infecciones de transmisión sexual, en general, se ha considerado bajo. Sin embargo en los últimos años, existe un número creciente de "mujeres mayores" que han comenzado ha encontrar nuevas parejas, a través de sitios de citas en línea (internet), y que al no ser el "grupo objetivo" y que al carecer de información, están dispuesta a tener relaciones sexuales sin protección, debido a esta situación, se produce una discusión ética con respecto a la segmentación en mercados sociales (Bateson, Weisberg, Mc Caffory, \& Juscombe, 2011). 
Otra aplicación que podemos señalar, son las campañas de marketing de las compañías tabacaleras, que muestran que las segmentación de marcas de cigarrillos, fueron desarrolladas para atender las necesidades psicológicas y psicosociales, dirigidos a consumidores para que se dificulte la posibilidad de dejar de fumar (Le Cook, Wayne, Keinthly, \& Connoly, 2003). En estos casos, mediante empleos de técnicas de segmentación de mercados para la comercialización social, los efectos indeseados de las campañas de las tabacaleras, pueden ser evitadas mediante la aplicación de la segmentación de esos grupos, por el Marketing Social. También hay que señalar, que existen diferentes segmentos, en relación con las respuestas a los mensajes contra el tabaquismo, que pueden variar en su respuesta, debido a su comprensión y tipos de mensajes, así como también por la respuesta en sí a una campaña en particular. En este sentido pueden haber grupos que muestren respuestas negativas (Walsh, Shiu, Graig Andrews, \& Hasting, 2010), debido a la inflexibilidad de los comportamientos grupales que suelen participar en el Marketing Social, y que podría ser explicado debido a componentes actitudinales como: negación e ira, conductas arraigadas y resistentes a las presiones de las tendencias sociales (Pechmann, Zhau, Goldberg, \& Reibling, 2003). Estas contradicciones de los resultados de las campañas en segmentos, sin embargo, pueden estar asociados a la visualización de los anuncios antitabaco y no a la segmentación.

En este contexto, los fumadores y los no fumadores por ejemplo son destinatarios de las campañas ¡Help!, sin embargo es probable que en estos dos grupos, sus respuestas sean muy diferentes, debido a sus puntos de vista y actitudes acerca de fumar (Walsh, Shiu, Graig Andrews, \& Hasting, 2010). Por ello es relevante señalar que el cambio de comportamiento, que debería alcanzarse a través de la campaña "Help", difieren entre estos dos grupos. Esto también puede ser debido al grado de responsabilidad social que tiene los grupos objetivos, es decir, la influencia de la cultura, las creencias y los valores enfrentados a la publicidad, estos son patrones de pensar, sentir y actuar, que tienen sus raíces en los valores comunes y en las convenciones sociales (Nakata \& Sivakumar, 2001). Además el público objetivo, a menudo no reconocen que tienen un problema, y mucho menos están en busca de una solución. Al respecto podemos señalar, que el Marketing Social ha empleado tradicionalmente estrategias, instrumentos $y$ técnicas tomadas de los modelos comerciales de marketing. Y las estrategias de segmentación, que son un éxito notable en los productos y servicios de marketing, a menudo producen resultados subóptimos en el Marketing Social. Dado que el objetivo del Marketing Social, es la creación de un cambio de comportamiento y sostenerlo en el tiempo, es necesario reexaminar y reinventar las modalidades que pueden provocar el cambio de comportamiento deseado (Smith, 2006).

Por esto las estrategias exitosas de segmentación, apuntar y posicionar en Marketing Social, se deben generalmente a estrategias de comunicación más personalizadas, las que claramente son más eficaces. La definición de las diferencias en los comportamientos, es una estrategia útil, en la elección apropiada de una estrategia de marketing social (Smith, 2006). "De hecho, como han señalado numerosos autores (Andreasen, 1995; Andreasen, 2006; Bloom y Novelli, 1981; Brenkert, 2002; Dholakia
1984), campañas de marketing social que utilizan la segmentación son comúnmente criticadas, por discriminar a grupos o poblaciones específicas, en particular por las agencias gubernamentales que financian estas campañas (Walsh, Shiu, Graig Andrews, \& Hasting, 2010). En consecuencia, existe un riesgo muy real, de que un método eficaz para mejorar la eficacia de las campañas de marketing social, no se puede utilizar en la mayoría de las veces (Newton, Newton, Turk, \& Ewing, 2013).

Al respecto, en la determinación de aplicación del SAP en Marketing Social, se pueden llevar a cabo evaluaciones, de la conciencia y la comprensión del mensaje retratado, así como también de la actitud hacia la campaña, por el grupo objetivo determinado. Es importante en este aspecto, la evaluación exante, los supuestos culturales de los puntos críticos de los grupos segmentados, siendo al respecto también clave: el valor cultural, las normas culturales del grupo dominante, los valores sociales, las creencias, las percepciones y/o actitudes. Los cuales crean diferencias en los patrones de comportamiento de estos grupos. Estos valores culturales distinguen y definen grupos culturales, y tienen un importante papel que desempeñar en el éxito de los programas de mercadeo social, sobre todo en las sociedades multiculturales (Smith, 2006). La comprensión de los valores de varias subculturas, su identidad étnica y el patrimonio cultural, se ha convertido en la clave del éxito de los programas de mercadeo social (Smith, 2006).

También es relevante, al respecto, medir los efectos, "si no lo puedes medir, mal lo puedes gestionar"Medir, medir, medir..... Se refiere a medir los comportamientos de compra del consumidor, para contar con mejores herramientas de gestión de Productos y Precios (Weinreich, La practica del marketing y su evolución en el tiempo, 2016). Algunas herramientas para medir y analizar son: Regresión Multivariable, Discriminant Analysis, Cluster Analysis, Factor Analysis, Multidimensional Scaling, Conjoint Analysis, Diseños Experimentales (Weinreich, La practica del marketing y su evolución en el tiempo, 2016). Además, hay un factor importante, que es la "participación", este principio considera la importancia de la participación activa de la audiencia y el control compartido, sobre el diseño y la ejecución de actividades específicas, en el marco de medición del Marketing Social.

En cuanto a la metodología de segmentación y posicionamiento, el método adecuado dependerá principalmente de los objetivos y estrategias, que tenga la comercialización social. Por ejemplo podría ser un objetivo de la comercialización social, comunicar claramente el mensaje a los distintos segmentos a los que se apunta, esperando que éstos lo entiendan con claridad, bajo el fin de lograr el cambio deseado del comportamiento (Smith, 2006). Dentro de las herramientas metodológicas, está la técnica de estudio antropológico de la etnografía (Smith, 2006), además estas variables son utilizadas en el diseño de las estrategias que acoge el marketing social. Así mismo, podemos señalar otras herramientas, como: los grupos focales, entrevistas en profundidad, encuestas personales. Las cuales pueden utilizarse para sondear a los sitemas de valores específicos, de la cultura de los dintintos segmentos de grupos elegidos. También como metodología complementaria, podemos citar, la busqueda de literatura secundaria, estudios y analisis bibliográficos, estudios de casos, 
etc. Al mismo tiempo, el Marketing Social debe basarse en modelos teóricos, que guían la selección de los determinantes más relevantes, como son: los grupos prioritarios; los objetivos; las intervenciones y las evaluaciones para el cambio de comportamiento a escala, las teorías de la difusión de las innovaciones, las redes sociales, la comunidad activa, la economía política y el capital social (Walsh, Shiu, Graig Andrews, \& Hasting, 2010).

Podemos citar por otra parte, que en el ámbito internacional, el éxito de la aplicación de segmentación social, se puede deber a que los mercados de grupos sociales y no los individuos, son su enfoque, para el análisis, la planificación y ejecución. Además los elementos de intervención social, se prueban previamente con el grupo objetivo, con diferentes variables de segmentación, que son considerados, al seleccionar la estrategia de intervención, donde el grupo objetivo de intervención se adapta para el segmento seleccionado (Walsh, Shiu, Graig Andrews, \& Hasting, 2010).

La estrategia de intervención, busca cambiar el comportamiento medible. Sin embargo, sin una actitud positiva, de parte de los grupos receptores, los cuales son los destinatarios de la publicidad social, estos grupos no estarán motivados para comprometerse con el mensaje y por lo tanto es poco probable, que se convenzan a modificar sus comportamientos. En este aspecto, varios estudios han puesto de relieve la importancia de la comprensión de los mensajes dirigidos, en términos de eficacia de la publicidad, por ello la comprensión, es el primer paso en el fenómeno de la persuasión (Jacoby \& Hoyer, 1989).

Una aplicación de intervención en Salud Pública, para segmentar utiliza por ejemplo el origen étnico, edad, u otros datos demográficos, como una base para la identificación de segmentos y subgrupos distintos, dentro del grupo social objetivo. O también dividiendo la población en diferentes segmentos sobre la base de las conductas y actitudes, la disposición al cambio, las intenciones futuras, la lealtad al producto o servicio y los aspectos psicográfico (Greir \& Bryant, 2005). Así mismo, la identificación de uno o más segmentos como el grupo objetivo, para recibir la mayor prioridad en el desarrollo del programa, con estrategias de marketing social diferenciados. En programas de intervención de salud pública, que inicialmente se segmenta su población objetivo por edad (jóvenes 9-13 años de edad y los padres influenciadores), al respecto, investigaciones han demostrado que se identifican diferencias importantes entre segmentos específicos, dentro de la audiencia sobre la base del nivel de actividad, la receptividad a la actividad física, etnia y género (Greir \& Bryant, 2005).

Así, la eficiencia de las campañas está en la adaptación de las estrategias, para hacer frente a las necesidades de los distintos segmentos. Aquí lo relevante es la aplicación con educación. Al respecto, la educación informa y convence a la gente a adoptar conductas saludables voluntariamente, dando a conocer los beneficios de cambiar. Como ejemplo, cuando los profesionales de la salud educan a la gente, acerca de los beneficios de adoptar hábitos de vida saludables, los ciudadanos tienen libertad de elección en cómo responder, y la sociedad acepta los costos, cuando algunas personas continúan practicando conductas indeseables. La educación es más eficaz cuando los objetivos de la sociedad son consistentes con los de la audiencia objetivo y los beneficios del cambio de comportamiento, entonces los costos de cambiar son bajos, las habilidades y otros recursos necesarios para cambiar son fácilmente disponibles (por ejemplo, poner a un bebé a dormir sobre su espalda para prevenir el síndrome de muerte súbita del lactante) (Rothschild, 1999). También, se ha demostrado que la legislación es la herramienta más eficaz para la salud pública, cuando la sociedad no está dispuesto a pagar los costos asociados, con la práctica continua de un comportamiento poco saludable o de riesgo. Por otra parte y al respecto, el Marketing Social influye en el comportamiento, ofreciendo opciones alternativas que invitan al intercambio voluntario.

Algunas críticas a la estructura de los programas de Marketing Social radican por ejemplo en que la segmentación del público en la salud pública, también está limitada por una dependencia excesiva de la etnia y otras variables demográficas, como también en las etapas de cambio, y que tiene una forma muy teórica (Prochaska \& Diclements, 1984). Andreasen, ha argumentado que el marketing social se está moviendo en un periodo de madurez temprana, con creciente popularidad entre los profesionales de la salud pública. Sin embargo, para continuar el desarrollo, el marketing social se debe superar una serie de retos. En materia de salud pública, estos desafíos se pueden agrupar en cuatro categorías: (a) los conceptos erróneos y otras barreras a la difusión, metodologías (b) investigación y evaluación formativa, (c) las cuestiones teóricas, y (d) las consideraciones éticas (Andreasen \& Kotler, 2003). Un problema recurrente es que, el marketing social es a menudo visto como un método para diseñar campañas de comunicación, en lugar de desarrollar intervenciones integrales, que realmente integren la mezcla de marketing completa de producto, precio, plaza y promoción (Greir \& Bryant, 2005).

En relación a lo anterior y en muchas ocasiones, la educación y la política son a menudo componentes de una aplicación e intervención de mercadeo social (Greir \& Bryant, 2005). Sin embargo, es la comercialización la que crea un entorno más propicio para el cambio, aumentar el atractivo de los beneficios ofrecidos y la minimización de los costos, y por ende una asignación más eficientes de los recursos.

En otro punto importante y en referencia a los beneficios que comprende el diseño de las campañas de Marketing Social, éstas satisfacen una motivación subyacentes de grupos de personas (segmentos), que a veces, no era a lo que apuntaba principalmente el objetivo de la campaña, como por ejemplo una campaña para mejorar la salud en ciudades capitales, subyace a cuestiones relacionas, como un medio ambiente más limpio o la satisfacción de los servicios a usuarios en la salud pública, etc. Al respecto generalmente son beneficios diferidos y distantes (Smith, 2006).

\section{Aplicación de la mezcla de Marketing (Producto, Precio, Plaza y Promoción) en Marketing Social}

LamezcladeMarketing, tambiénconocidocomolas4P: producto, precio, plaza y promoción. Estos elementos también se consideran importantes en Marketing Social y son fundamentales para la planificación y ejecución de una estrategia de marketing integrado (Greir \& Bryant, 2005). El Producto, se 
refiere al conjunto de los beneficios asociados con el comportamiento deseado o el uso del servicio, y distingue entre el producto principal (lo que la gente va a ganar cuando se realiza el comportamiento) y el producto real (el comportamiento deseado), también se utiliza el concepto del producto aumentado, para referirse a cualquier objeto y servicios tangibles, usados para facilitar el cambio de comportamiento (Kotler, Robert, \& Lee, Social Marketing: Improving The Quality of life, 2002).

"El Precio se refiere al costo o sacrificio intercambiados, por los beneficios prometidos, este costo se considera siempre desde el punto de vista del consumidor. Como tal, el precio por lo general abarca los costos intangibles, como el placer disminuido, la vergüenza, la pérdida de tiempo y la molestia psicológica que a menudo acompaña al cambio, especialmente cuando la modificación de los hábitos están arraigados. Al establecer el precio correcto, es importante saber, si los consumidores prefieren pagar más para obtener el valor agregado de beneficios y si creen que los productos regalados o de bajo precio son inferiores a los más caros" (Greir \& Bryant, 2005, p. 323)

El lugar o Plaza se refiere a la distribución de bienes y la ubicación de las ventas y los encuentros de servicio (Kotler \& Armrstrong, Marketing Versión para Latinoamerica, 2007). En el marketing social, puede ser pensado como puntos de acción: donde y cuando el mercado de destino, llevará a cabo el comportamiento deseado, podrá adquirir objetos tangibles relacionados, y recibir cualquier servicio asociado. "El Lugar incluye la ubicación física real de estos puntos de venta, horas de funcionamiento, el atractivo general y confort, accesibilidad y por ejemplo, la disponibilidad de estacionamiento y del transporte público. También incluye intermediarios, organizaciones y personas, que puede proporcionar información, bienes y servicios, y realizar otras funciones que facilitan el proceso de cambio" (Greir \& Bryant, 2005, p. 323).

La promoción comúnmente es el componente más perceptible de la comercialización, la promoción contiene por ejemplo: el tipo de vendedores de capacidades comunicativas persuasivas. Además que se utilizan para transmitir los beneficios del producto y los objetos tangibles asociados, los servicios, las estrategias de precios, y los componentes de lugar. Las estrategia de promoción consiste en un conjunto de actividades ( relaciones públicas, materiales impresos, artículos de promoción, señalización, eventos especiales y exposiciones, la venta cara a cara, y los medios de entretenimiento) cuidadosamente diseñadas, destinadas a influir en el cambio social y por lo general implica varios elementos, como: los objetivos específicos de comunicación, para cada público objetivo o grupo social segmentado; directrices para el diseño de llamar la atención; eficaces mensajes; y la designación de los canales de comunicación apropiados (Greir \& Bryant, 2005). En relación a lo anterior, podemos citar que el peligro de que un énfasis excesivo, en la traducción directa de los principios y prácticas de marketing comercial, y que se los incorpore a contextos sociales, puede crear problemas prácticos y confusión con respecto a la base teórica del Marketing Social. Por ejemplo, con respecto a la teoría de los programas de mercadeo social, se recomiendan que las $4 \mathrm{P}$, se vuelven a conceptualizar como por ejemplo: proposición social (producto) y costos (precio) (Peattie \& Peattie, 2003).

Entonces, y en relación a lo expuesto el Marketing Social, tiene como objetivo, el cambio de comportamiento agregado, y para ello utiliza segmentos prioritarios de la población, y por esto la individualidad no es el centro de los programas, sino los grupos sociales.

\section{Algunas experiencias de Empresas en Marketing Social, aplicando SAP en Chile}

La siguiente tabla № 1 , presenta algunos casos de empresas que aplican el marketing social, utilizando S.A.P. en Chile.

\begin{tabular}{|c|c|}
\hline Enami Ventanas & $\begin{array}{l}\text { Campañas de promoción con orientación centrada en las personas, de poblaciones circundantes a la planta, promocionando el trabajo en equipo, } \\
\text { el control de riesgos, control de calidad y gestión ambiental. La responsabilidad social aplicada al Marketing, en esta empresa, permitió una alianza } \\
\text { estratégica, desarrollar la participación, y el valor y el compromiso. Lo que sirvió finalmente para que Codelco comprara esta empresa en el } 2005 \\
\text { (Bravo, Responsabilidad Social RS La nueva riqueza de las naciones, 2007). }\end{array}$ \\
\hline Banco Estado & $\begin{array}{l}\text { Proyecto de RSE y Marketing Social de Bancarización, es decir llevar los servicios financieros a poblaciones marginales. Garantizar los servicios } \\
\text { financieros a sectores productivos, que por razones de aislamiento, riesgo o rentabilidad, no son adecuadamente atendidos por la banca privada, } \\
\text { campaña dirigida al segmento de ingresos bajos y poblaciones marginales. (Teixidó, Chavarri, \& Castro, 20o2). }\end{array}$ \\
\hline Ambrosoli & $\begin{array}{l}\text { Campañas de Marketing Social, dirigida al rescate de los valores tradicionales, tales como: disciplina, orientación total al cliente, calidez y respeto } \\
\text { a la jerarquía, importancia de la familia y la organización. La visión inculcada por uno de sus dueños Constantino Ambrosoli “Que la empresa } \\
\text { actuara al más alto nivel social" (Bravo, Responsabilidad Social RS La nueva riqueza de las naciones, 2007) }\end{array}$ \\
\hline $\begin{array}{l}\text { Compañía Siderúrgica } \\
\text { Huachipato S.A. }\end{array}$ & $\begin{array}{l}\text { Promoción de la política de cumplimiento medioambiental, en este punto se trabaja, se segmenta y se apunta al conjunto de la comunidad y de las } \\
\text { autoridades; también apuntar a las relaciones con los clientes y proveedores y al trabajo tripartita con la comunidad-municipio-empresa. }\end{array}$ \\
\hline Masisa Chile & $\begin{array}{l}\text { Promoción en los medios de su filosofía, "como una filosofía distinta de lo tradicional para hacer negocios, definen en Masisa Chile a la política } \\
\text { institucional de Responsabilidad Social y ambiental, un fuerte apoyo a la capacitación del personal y sus familiares, la preocupación por la salud de } \\
\text { los trabajadores, los programas pioneros de formación de microempresarios mueblistas y un modelo de gestión ambiental responsable y proactivo" } \\
\text { Programa que apunta a los clientes constructores, empresas y personas individuales (Financiero, 2006). }\end{array}$ \\
\hline Gerdau AZA & $\begin{array}{l}\text { Aplicación de marketing Social con su orientación al cliente y su compromiso con la sustentabilidad, lo han hecho merecedor del premio al mejor } \\
\text { reporte de sustentabilidad 2007 (EMB, 2006) }\end{array}$ \\
\hline $\begin{array}{l}\text { DOLE (Dole Food } \\
\text { Company) }\end{array}$ & $\begin{array}{l}\text { Marketing Social, apuntando al desarrollo de principios y acciones de Negocio Responsable, el cual involucra temáticas medioambientales, } \\
\text { relación con los trabajadores, calidad de los productos, relaciones con la comunidad y su campaña antidroga. Destaca haber recibido la certificación } \\
\text { ISO } 14 . \text { oo1 en Chile, la cual incluye la verificación de los procesos de cultivo, proceso de packing y conservación de fruta fresca; fueron incluidos en el } \\
\text { proceso a 6o productores de fruta externos a la compañía. Además la empresa filial chilena ‘Cartones' fue certificada el año } 2000 \text { en ISO 9.001, esta } \\
\text { empresa fabrica cajas de cartón para la exportación de fruta fresca. (Tendencias de la Responsabilidad Social Empresarial de paises en vias de } \\
\text { desarrollo, 2014) }\end{array}$ \\
\hline
\end{tabular}




\begin{tabular}{|c|c|}
\hline $\begin{array}{l}\text { DEL MONTE FRESH } \\
\text { PRODUCE S.A. }\end{array}$ & $\begin{array}{l}\text { En su web valora la ética de los negocios, Campañas de promoción donde se relaciona con el uso de pesticidas y la inocuidad alimentaria.. } \\
\text { (Tendencias de la Responsabilidad Social Empresarial de paises en vias de desarrollo, 2014) }\end{array}$ \\
\hline XEROX CHILE & $\begin{array}{l}\text { Marketing Social, con campañas internas, diseñadas a promocionar la ética empresarial y códigos de conducta, dirigido a sus propios trabajadores } \\
\text { y a las empresas que atiende, con campañas como: NO discriminar, NO al acoso sexual, igualdad de oportunidades, seguridad en el trabajo, } \\
\text { capacitación. En la promoción de Responsabilidad Social Empresarial externa, el cuidado del medio ambiente (Quezada \& Sánchez, 2009). }\end{array}$ \\
\hline $\begin{array}{l}\text { Coca-Cola Embonor } \\
\text { Chile }\end{array}$ & $\begin{array}{l}\text { Promoción y programas de medio ambiente limpio, por ejemplo través del reciclaje, dirigido a sus clientes jóvenes. Y el desarrollo del } \\
\text { Marketing Responsable. }\end{array}$ \\
\hline $\begin{array}{l}\text { BCI, Banco de Crédito } \\
\text { e Inversiones }\end{array}$ & $\begin{array}{l}\text { A partir del } 2006 \text { se establece un programas de Marketing Social, en favor del medio ambiente, cómo son: talleres; programas de arborización de } \\
\text { plazas; ahorro de luz, agua y papel en todas las sucursales; programas de conciencia en el uso de recursos renovables y no renovables, apuntando al } \\
\text { segmento de potenciales clientes. (Quezada \& Sánchez, 20o9). Mensajes cómo “Despierta” dirigido a los clientes, niños, jóvenes, adultos y tercera } \\
\text { edad, a cuidar el medio ambiente, porque todavía hay mucho que hacer. }\end{array}$ \\
\hline Viña Concha y Toro & $\begin{array}{l}\text { A través de esta política, la empresa orienta sus esfuerzos y canaliza sus aportes hacia proyectos que contribuyan a crear valor para la sociedad y el } \\
\text { entorno donde opera. Promoción de consumo responsable de sus vinos. }\end{array}$ \\
\hline CMPCS.A. & $\begin{array}{l}\text { Campañas de Marketing Social, que promociona la cultura, el desarrollo social y del medio ambiente. Programas de promoción de la Responsabilidad } \\
\text { Social interna con los trabajadores. Además de Programas educacionales, dirigidos al segmento de los grupos sociales adyacentes a su empresas. } \\
\text { (Teixidó, Chavarri, \& Castro, 2002). }\end{array}$ \\
\hline CCU CHILE & $\begin{array}{l}\text { Campañas de Marketing Social, como: “Educar el consumo responsable de alcohol”, dirigido a todos sus clientes, apunta hacia los adolescentes y } \\
\text { además de estar siempre en sintonía con cada uno de los públicos, a los que apuntan sus productos. } \\
\text { Campañas dirigido a la comunidad como: “Campaña fiestas patrias”; “Año nuevo”; “Si vas a beber pasa las llaves”, y campañas viales junto a } \\
\text { Carabineros de Chile. } \\
\text { Campañas "verano Responsable”, dirigido a los jóvenes y adultos que veranean en playas de la costa Chilena, "verano con Responsabilidad” “ Pásalo } \\
\text { bien con Responsabilidad” } \\
\text { Dirigido a sus clientes (negocios), campañas como: "No venta de alcohol a menores”; “Disfruta tú edad”; } \\
\text { Dirigido a sus trabajadores, campañas de marketing social interno, como: “Educar en familia”, "Capacitación Interna” (Quezada \& Sánchez, 20og). }\end{array}$ \\
\hline Forestal Millalemu & $\begin{array}{l}\text { Campañas de promoción de sus formas de hacer las cosas, hacia la comunidad, cómo informar sobre su certificación ISO 140oo y ISO } 14001 . \\
\text { Relaciones con la comunidad externa, certificación FSC. Programas de promoción y apoyo a la comunidad, como apoyo a la forestación campesina. } \\
\text { Promoción del Proyecto san Isidro, es un centro de capacitación para trabajadores y la comunidad de cabrero en general, replicando la experiencia de } \\
\text { INFOCAP del Hogar de Cristo. Misión Eco eficiencia de la empresa. (Teixidó, Chavarri, \& Castro, 2002). }\end{array}$ \\
\hline Minera Escondida & $\begin{array}{l}\text { Campañas de promoción de su fundación Educacional Escondida, centro educacional de capacitación técnica. Relaciones laborales y extensión a la } \\
\text { comunidad. Convenio con universidades locales, programas de medio ambiente. }\end{array}$ \\
\hline
\end{tabular}

\section{Algunas experiencias de programas La siguiente tabla № 2 , expone las experiencias internacionales y nacionales de de algunos programas de marketing social, Marketing Social y SAP especialmente en el ámbito de la salud y educación pública, utilizando SAP (segmentar, apuntar y posicionar).}

Tabla 2 Experiencias internacionales y nacionales de programas de Marketing Social y SAP

\begin{tabular}{|c|c|}
\hline Programa & Experiencia Marketing Social \\
\hline Road Crew & $\begin{array}{l}\text { Marketing Social, mediante la Intervención, en un esfuerzo por frenar los accidentes automovilísticos relacionados con el alcohol, este programa } \\
\text { está dirigido a hombres de entre } 21 \text { y } 34 \text { años de edad que conducen solos a casa, después de una noche de beber en las tabernas en Wisconsin rural. } \\
\text { Los diseñadores del programa crearon un servicio de viaje, que transportó a los hombres de sus casas a los bares, entre bares, y de vuelta a casa de } \\
\text { nuevo, lo que les permite disfrutar de su noche sin riesgo de conducir en estado de ebriedad. } \\
\text { Una agencia de publicidad desarrolló el nombre del programa (del equipo del camino), lema y logotipo. Al final del primer año, los resultados de la } \\
\text { evaluación, indican que el programa ha disminuido los accidentes relacionados con el alcohol en un 17\%. (Creir \& Bryant, 2005). }\end{array}$ \\
\hline WIC de Texas & $\begin{array}{l}\text { Programa de mercadeo social, llevado a cabo para aumentar la matrícula y mejorar la satisfacción del cliente y del empleado, con el Programa } \\
\text { Especial de Nutrición Suplementaria para Mujeres, Infantes y Niños (WIC) en Texas. EL SAP se logro mediante entrevistas, observación } \\
\text { participante, en profundidad, entrevistas telefónicas, grupos de enfoque y encuestas, que se utilizaron para entender las necesidades, preferencias } \\
\text { y características de las cuatro audiencias objetivo: familias elegibles pero que no participan en el programa, los participantes del programa, los } \\
\text { empleados del programa, y los profesionales que referir a las personas con el programa. Los resultados se utilizaron para desarrollar un plan de } \\
\text { marketing social integral, que incluía cambios en las políticas, mejoras en la prestación de servicios, el personal y la formación de proveedores, } \\
\text { promoción interna, la información pública y las comunicaciones, la educación del cliente, y las intervenciones basadas en la comunidad. } \\
\text { Este plan funcionó para cambiar la percepción de las familias de que WIC era un programa de bienestar, que proporciona comida gratis a los pobres, } \\
\text { haciendo hincapié ahora en la educación sobre nutrición, chequeos de salud, inmunizaciones, y referencias, ayudando a las familias, ayudarse a } \\
\text { sí mismos. En el que las familias puedan mantener su orgullo y autoestima, a medida que ganan sus beneficios de WIC y aprender acerca nutrición } \\
\text { yotras formas de ayudar a sus propias familias (Greir \& Bryant, 2005). }\end{array}$ \\
\hline The Food Trust & $\begin{array}{l}\text { Food Trust, es una organización sin fines de lucro en PhiladelPhia, Pennsylvania, cuyo objetivo es aumentar el acceso de las personas a alimentos } \\
\text { asequibles y nutritivos. La mejora de las opciones de alimentos para los jóvenes, en tiendas de locales de barrio. La campaña el marketing social } \\
\text { promueve la demanda de aperitivos saludables, promover la participación de los estudiantes en los programas de alimentación escolar, y también } \\
\text { apuntar a la industria alimentaria, para aumentar la disponibilidad de opciones de alimentos saludables en las tiendas locales. } \\
\text { Por ejemplo, la Food Trust ha desarrollado alianzas con empresas de aperitivo individuales, para aumentar la distribución de las opciones más } \\
\text { saludables en las tiendas de barrio. La investigación formativa también se llevó a cabo con jóvenes entre las edades de 5-12 años, para entender su } \\
\text { comportamiento con bocadillos y cómo promover mejor las opciones de bocadillos saludables, disponibles en la actualidad (Creir \& Bryant, 2005). }\end{array}$ \\
\hline $\begin{array}{l}\text { Ministerio de Salud } \\
\text { Chile (Minsal) }\end{array}$ & $\begin{array}{l}\text { Desarrollo de campañas de prevención del SIDA (a través de promover el sexo seguro, dirigido a adolescentes y adultos), campañas anti influenza } \\
\text { (a través de campañas de vacunación temprana), campañas contra la meningitis en niños y adolescentes y la también la campaña antitabaco } \\
\text { (con promoción explicativa , basado en datos empíricos de lo dañino que es fumar, promoción a través de la muestra de experiencias, logotipos } \\
\text { y fotos en las cajetillas de cigarro) (Minsal, 2016). }\end{array}$ \\
\hline
\end{tabular}

Fuente: Elaboración propia, basado en literatura examinada. 


\section{Conclusiones}

En general se observa un contexto teórico dinámico y evolutivo en lo que respecta al Marketing Social. Lo que se inició como un concepto de Marketing acotado y de dependencia del marketing comercial, se fue ampliando, y ha evolucionado hacia un sistema estructurado, extenso e integral, modelado y de amplias perspectivas de aplicación, en las diversas sociedades, donde este proceso se ha podido observar. Por lo que el marketing social, es un proceso integral social, para lograr un cambio en el comportamiento público a escala, de los grupos de personas intervenidas.

Ha sido interesante también observar, que una parte del desarrollo del Marketing Social, se ha focalizado en los temas de la sustentabilidad ambiental, social, cultural, y de la satisfacción con valor, seguido de las implicaciones que dichas intervenciones tiene sobre el comportamiento ético de los actores sociales, de sus conductores y de la sociedad como un todo.

Podemos señalar que el marketing social, se convirtió en un proceso que abarca las organizaciones privadas con fines y sin fines de lucro, pero también las entidades públicas, las que sistemáticamente han incorporado el Marketing social en la gestión estratégica de las organizaciones, en la gestión corporativa y en la dirección.

En la actualidad, las evidencias de estudio y aplicación de marketing social y del SAP, se pueden observar experiencias a nivel de empresas privadas de diversa naturaleza, que han aplicado el proceso de Marketing Social, detallado en la tabla № 1, con campañas dirigidas al beneficio social de los grupos objetivo, con respuestas de éstos en forma variadas. Se aprecia una aplicación de SAP no completa en referencia al marketing social. También podemos exponer programas (tabla №2), tanto a nivel nacional como internacional de marketing en salud pública, enfocados en la prevención y la educación de los grupos sociales. Determinando procesos de intervención del marketing social a escala y que también es una herramienta útil para la orientación estratégica de los gestores de la salud pública. También hay que mencionar que a través de las experiencias vistas, se muestra la eficacia y la limitación de las materias sociales para hacer frente a un cambio de comportamiento a gran escala.

En relación a las aplicaciones de marketing Social y S.A.P., podemos señalar que si bien es cierto han sido efectivas en general, principalmente las relacionadas con la salud pública, educación, política y sociedad. Sin embargo se aprecian contradicciones en los resultados, los cuales pueden deberse a distorsiones en la comprensión del mensaje o debido a problemas actitudinales del receptor, así como también por la ocurrencia en que grupos sociales no objetivo quedan afuera de las campañas y a veces ellos también se podrían beneficiar socialmente de éstas, produciéndose entonces una discusión ética del proceso de segmentación en el marketing social.

En chile se manifiesta un lento pero persistente interés y preocupación por el tema del marketing social y su medición, sin embargo en la actualidad se manifiesta como un proceso en marcha sostenida.
Internacionalmente está bastante integrado a las estrategias y tácticas de organizaciones privadas y públicas, las cuales obtienen resultados eficaces en este proceso de mercadeo social.

\section{Referencias}

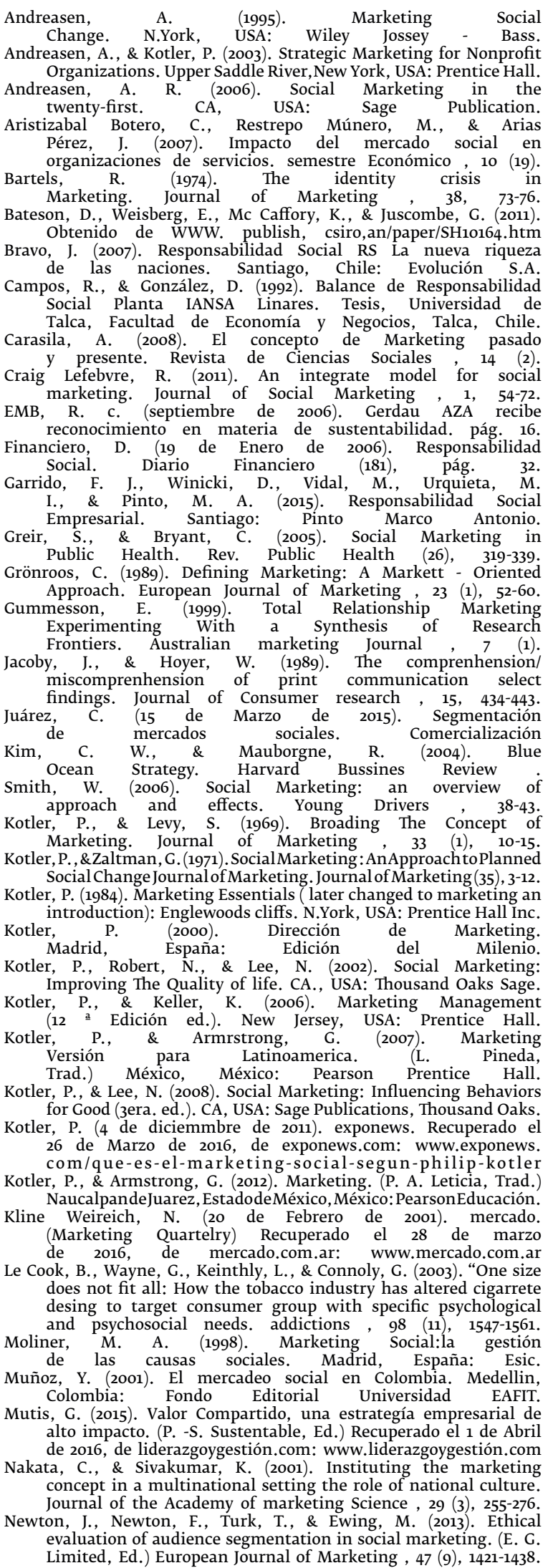


Pechmann, C., Zhau, G., Goldberg, M., \& Reibling, E. (2003). What to convey in anti-smoking sdvertisements of adolescents the use of protection motivation theory to identify effective message themes. Journal of marketing , 67 (2), 1-18. Programas de salud pública, (2016). minsal. 'Recuperado el 10 de Abril de 2016, de minsal.cl: http.//www.minsal.cl Quezada, D., \& Sánchez, M. (2009). Impacto de la RSE en los consumidores Chilenos. Tesis, Universidad de las Américas, Facultad de Ciencias Ecnómicas y Administrativas, Santiago.

Rayport, J., \& Sviokla, J. (1995). Exploting The Virtual Value Chain. Harvard Business
Raval, Deview , 73 (12), $75-87$. driven segmentation in social marketing. Journal of driven segmentation in social marketing. Journal of

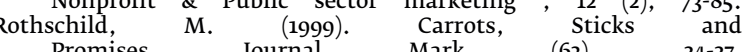
Promises. Journal Mark (63)

$24-27$. , E., \& Tamilia, R. (2001). Robert Bartels and The History of Smith, Marketing Thoght. Journal of Macromarketing , 21 (2), 156-163. approach and effects. Young Drivers, 38-43. Stanton, W.
Marketing. N.York, USA: Mc Graw ${ }^{\text {(1969). }}$ Hill.
Lazer, W. (1969). Marketing`s Changing Social Relationships. Journal of Marketing , 33 (1), 3-9. Suárez Lugo, N. (2013). Social Marketing as a supporting tool for sexual health in Cuba. Revista Cubana de la Salud Pública , 39. Tendencias de la Responsabilidad Social Empresarial de paises en vias de desarrollo, c. C. (2014). Vincular. Recuperado el 7 de Enero de 2015, de Vincular.org: http.//www.vincular.org Teixidó, S., Chavarri, R., \& Castro, A. (2002). Responsabilidad Social, 12 casos empresariales en Chile. Fundación Prohumana. Santiago: Fundación Prohumana

Walsh, G. H., Shiu, E., Graig Andrews, J., \& Hasting, G. (2010). segmentation in Social Marketing: Insights From The Europen Union`s multi-country. European Journal of Marketing , 44, 1140-1164. Weinreich, R. (Mayo de 2007). S.A.P. : Segmentar, Apuntar, Posicionar La nueva tónica del marketing de hoy. Trend Management , 56-62. Weinreich, R. (19 de Marzo de 2016). Segmentación de Mercados. Conceptos, propósitos y metodología. Santiago, Chile.

Weinreich, R. (2016). Value Management: La Gestión Estratégica de Posicionamiento de Productos ( GEPP). Santiago, Chile.

Weinreich, R. (2016). La practica del marketing y 